\title{
The Structure of Higher Degree Symmetry Classes of Tensors*
}

\section{Russell Merris**}

(March 22, 1976)

\begin{abstract}
The paper is concerned with symmetry classes of tensors which arise from a permutation group $G$ and irreducible character $\chi$ of $G$. In case $\chi$ is of degree 1, a well-known algorithm is available for inducing a basis of the symmetry class from the underlying vector space. When the degree of $\chi$ is greater than 1, no comparable construction has been discovered. The difficulties are discussed and results obtained in some special cases.
\end{abstract}

Key words: Decomposable (or pure) tensor products; irreducible complex character; orthogonality relations; permutation group.

\section{Introduction}

Let $V$ be a complex inner product space of dimension $n$. Let $\stackrel{m}{\otimes} V$ denote the $m$ th tensor power of $V$, and let $v_{1} \otimes \ldots \otimes v_{m}$ be the (pure or decomposable) tensor product of the indicated vectors. The inner product in $V$ induces an inner product in $\stackrel{m}{\otimes} V$ which is completely determined by its action on the set of decomposable tensors, namely

$$
\left(v_{1} \otimes \ldots \otimes v_{m}, w_{1} \otimes \ldots \otimes w_{m}\right)=\prod_{t=1}^{m}\left(v_{t}, w_{t}\right)
$$

By $S_{m}$, we mean the full symmetric permutation group on $\{1, \ldots, m\}$. If $\sigma \in S_{m}$, there is a (unique) linear operator $P\left(\sigma^{-1}\right)$ on $\oiint^{\sharp} V$ which has the effect $P\left(\sigma^{-1}\right) v_{1} \otimes \ldots \otimes v_{\mathrm{m}}=v_{\sigma(1)} \otimes \ldots \otimes v_{\sigma(m)}$, for all $v_{1}, \ldots, v_{m} \in V$. It follows that $P(\sigma) P(\pi)=\mathrm{P}(\sigma \pi)$. Moreover, from (1), $P(\sigma)^{*}=P\left(\sigma^{-1}\right)$. Let $G$ be a subgroup of $S_{m}$, and $\chi$ an irreducible (complex) character of $G$. Define

$$
T(G, \chi)=\frac{\chi(i d)}{\mathrm{o}(G)} \sum_{\sigma \in G} \chi(\sigma) P(\sigma),
$$

where $i d=$ identity of $G$, and $o(G)$ is the order of $G$. By the orthogonality relations for characters, $T(G, \chi)$ is an orthogonal projection onto its range $V_{\chi}(G)$ (see, e.g., [5] ${ }^{1}$ or [12]). The subspace $V_{\chi}(G)$ is called a symmetry class of tensors [8]. Several authors have exploited these symmetry classes to obtain information about so called generalized matrix functions (see, e.g., [5], [8], [9], and [11]).

Until recently, however, most of the work has involved only linear characters. One reason for this preference is the existence, in the case $\chi(i d)=1$, of a convenient basis for $V_{\chi}(G)$ which is induced from a given basis of $V$. In the case $\chi(i d)>1$, it is not easy to obtain such a basis. A more precise idea of our interest must await further introductory material.

With $\Gamma_{m, n}$ we denote the set of functions from the first $m$ positive integers to the first $n$. It is convenient to think of $\Gamma_{m, n}$ as a set of integer sequences of length $m$. Thus

AMS Subject Classification: Primary, 15A69; Secondary, 20C 15.

*An invited paper. The research leading to this article was supported in part by the National Science Foundation.

**Present address: Department of Mathematics, California State University, Hayward, CA 94542. 


$$
\Gamma_{m, n}=\{\gamma=(\gamma(1), \ldots, \gamma(m)): \quad 1 \leq \gamma(t) \leq n, \quad 1 \leq t \leq m\}
$$

If $e_{1}, \ldots, e_{n}$ is an orthonormal basis of $V$, it is well known (see, e.g., [8]) that $\left\{e^{\otimes}=\right.$ $\left.e_{\gamma(i)} \otimes \ldots \otimes e_{\gamma(m)}: \gamma \in \Gamma_{m, m}\right\}$ is an o.n. basis of $\otimes V$. It follows that $\left\{e_{\gamma}^{*}=T(G, \chi) e^{\otimes}: \gamma \in \Gamma_{m, n}\right\}$ must span $V_{\chi}(G)$. (In general, write $x_{1}{ }^{*} \ldots{ }^{*} v_{m}=T(G, \chi) v_{1} \otimes \ldots \otimes v_{m}$.) If $\alpha, \beta \in \Gamma_{m . n}$, observe that

$$
\begin{aligned}
\left(e_{\alpha}^{*}, e_{\beta}^{*}\right) & =\left(T(G, \chi) e_{\alpha}^{\otimes}, T(G, \chi) e_{\beta}^{\otimes}\right) \\
& =\left(T(G, \chi) e_{\alpha}^{\otimes}, e_{\beta}^{\otimes}\right) \\
& =\frac{\chi(i d)}{\mathrm{o}(G)} \sum_{\sigma \in G} \chi\left(\sigma^{-1}\right) \prod_{t=1}^{m}\left(\mathrm{e}_{\alpha \sigma(t)}, \mathrm{e}_{\beta(t)}\right) .
\end{aligned}
$$

It follows from (2) that $\left(e_{\alpha}^{*}, e_{\beta}^{*}\right)=0$ unless there is a $\pi \epsilon G$ such that $\beta=\alpha \pi$. We will say that $\alpha \equiv$ $\beta(\bmod G)$ if there exists a $\pi \epsilon G$ such that $\beta=\alpha \pi$. Clearly, " $\equiv(\bmod G) "$ is an equivalence relation.

If $\beta=\alpha \pi$, for some fixed $\pi \epsilon G$, then

$$
\begin{aligned}
\left(e_{\alpha}^{*}, e_{\beta}^{*}\right) & =\frac{\chi(i d)}{\mathrm{o}(G)} \sum_{\sigma \in G} \chi(\sigma) \prod_{t=1}^{m}\left(e_{\alpha \alpha(t)}, e_{\alpha \pi \sigma(t)}\right) \\
& =\frac{\chi(i d)}{\mathrm{o}(G)} \sum_{\tau \in G} \chi\left(\pi^{-1} \tau\right) \prod_{t=1}^{m}\left(e_{\alpha(t)}, e_{\alpha \tau(t)}\right) \\
& =\frac{\chi(i d)}{\mathrm{o}(G)} \sum_{\tau \in G_{\alpha}} \chi\left(\pi^{-1} \tau\right)
\end{aligned}
$$

where $G_{\alpha}=\{\tau \in G: \quad \alpha \tau=\alpha\}$ is the stabilizer subgroup of $\alpha$. In particular, by taking $\pi=i d$ in (3) one sees that $e_{\alpha}^{*} \neq 0$, if and only if

$$
\alpha \epsilon \Omega=\left\{\gamma \in \Gamma_{m, n}: \quad \sum_{\sigma \in \mathrm{G} \gamma} \chi(\sigma) \neq 0\right\}
$$

i.e., $\Omega$ consists of those sequences $\gamma$ which have the property that the restriction of $\chi$ to $G_{\gamma}$ contains the identically 1 character as a component. (Although not explicit in the notation, $\Omega$ depends on $m$, $n, G$ and $\chi$.) It follows that $\left\{e_{\omega}^{*}: \omega \epsilon \Omega\right\}$ spans $V_{\chi}(G)$.

Now, if $\alpha \equiv \beta(\bmod G)$, then $G_{\alpha}$ is conjugate to $G_{\beta}$. Therefore, $\Omega$ is a union of equivalence classes, i.e., if $\alpha \equiv \beta(\bmod G)$, then $e_{\alpha}^{*}=0$ if and only if $e_{\beta}^{*}=0$. Let $\bar{\Delta}$ be a system of distinct representatives for the equivalence classes in $\Omega$. (In practice, $\bar{\Delta}$ is usually chosen to consist of those elements of $\Omega$ which come first, in lexicographic order, in their equivalence classes.) Then

$$
\Omega=\underset{\alpha \in \bar{\Delta}}{\cup}\{\alpha \sigma: \quad \sigma \in G\}
$$

Theorem A ([10]): Let $\mathrm{e}_{1}, \ldots, \mathrm{e}_{\mathrm{n}}$ be a basis of $\mathrm{V}$. Then $\mathrm{V}_{x}(\mathrm{G})$ is the direct sum of the spaces $\left\langle\mathrm{e}_{\alpha \sigma}^{*}: \quad \sigma \in \mathrm{G}\right\rangle$, as $\alpha$ ranges over $\bar{\Delta}$. (The angular brackets denote linear closure.)

Proof: Choose the inner product on $V$ with respect to which $e_{1}, \ldots, e_{n}$ is orthonormal. The theorem follows from (4) and the definitions.

The result which makes the degree one case so fruitful is this:

Theorem B (Marcus and Minc [9]): Let $\mathrm{e}_{1}, \ldots, \mathrm{e}_{\mathrm{n}}$ be a basis of V. Suppose $\chi(\mathrm{id})=1$. Then $\left\{\mathrm{e}_{\alpha}^{*}: \quad \alpha \in \bar{\Delta}\right\}$ is a basis of $\mathrm{V}_{\chi}(\mathrm{G})$. 
Proof: It is routine to verify that $P(\sigma)$ commutes with $T(G, \chi)$ for all $\sigma \epsilon G$. Moreover, if $\chi($ id $)$ $=1$, then $P(\sigma) T(G, \chi)=\chi\left(\sigma^{-1}\right) \mathrm{T}(G, \chi)$. It follows that $e_{\alpha \sigma}^{*}=\chi(\sigma) e_{\alpha}^{*}$ if $\chi(i d)=1$. So, each subspace in the direct sum of Theorem $\mathrm{A}$ is one dimensional.

That $\left\{e_{\alpha}^{*}: \quad \alpha \epsilon \bar{\Delta}\right\}$ is not a basis of $V_{\chi}(G)$ when $\chi(i d)>1$ is evident from the following result of S. Pierce [12]:

Theorem C: Let $\alpha \in \bar{\Delta}$ be arbitrary. There is a $\sigma \in \mathrm{G}$ such that $e_{\alpha}^{*}$ and $\mathrm{e}_{\alpha \sigma}^{*}$ are linearly independent if and only if $\chi(\mathrm{id})>1$.

R. Freese [5] has improved Theorem C. Let $s_{\alpha}=\operatorname{dim}<e_{\alpha \sigma}^{*}: \quad \sigma \epsilon G>$. Freese's result is this:

THEOREM D. If $\alpha \in \Gamma_{m, n}$, then

$$
\mathrm{s}_{\alpha}=\chi(\mathrm{id})(\chi, \mathrm{l})_{\mathrm{G} \alpha},
$$

i.e., $\mathrm{s}_{\alpha}$ is $\chi(\mathrm{id})$ times the number of occurrences of the identically one character in the restriction of $\chi$ to $\mathrm{G}_{\alpha}$.

To conclude this section, we list a number of facts about $s_{\alpha}$ which follow from our discussion above.

(i) $s_{\alpha} \neq 0, \quad$ if and only if $\alpha \in \Omega$.

(ii) $\sum_{\alpha \in \Delta} s_{\alpha}=\operatorname{dim} V_{\chi}(G)$

(iii) $\chi($ id $) \leq s_{\alpha}<\chi(i d)^{2}$, for all $\alpha \in \Omega$.

(iv) $s_{\alpha} \leq\left[G: G_{\alpha}\right]$, for all $\alpha$. (In fact, it is clear from Freese's proof of Theorem D that $s_{\alpha}<\left[G: G_{\alpha}\right]$ unless $\chi$ is identically 1 and $G=G_{\alpha}$.)

(v) $\left\|e_{\alpha}^{*}\right\|^{2}=s_{\alpha} /\left[G: G_{\alpha}\right], \quad$ if $e_{1}, \ldots, e_{n}$ is an o.n. basis of $V$. (See (3).)

\section{Results}

Presently, the outstanding problem is to choose from $\left\{e_{\alpha \sigma}^{*}: \sigma \epsilon G\right\}$ a basis of $\left\langle e_{\alpha \sigma}^{*}: \sigma \epsilon G\right\rangle$. In this generality, the task seems quite difficult. We are able to supply an answer (Theorem 4 below) only in a very special situation.

As a first step toward analyzing the dependence relations among the elements of $\left\{e_{\alpha \sigma}^{*}: \sigma \in G\right\}, \alpha \in \Omega$, one is naturally led to consider

$$
G^{\alpha}=\left\{\sigma \epsilon G: \text { there exists } c_{\alpha}(\sigma) \text { such that } e_{\alpha \sigma}^{*}=c_{\alpha}(\sigma) e_{\alpha}^{*}\right\}
$$

(If $\chi(i d)=1$, then $G^{\alpha}=G$ and $c_{\alpha}=\chi$. Moreover, $G, \subseteq G^{\alpha}$ for all $\alpha \epsilon \Omega$.)

We first claim that $G^{\alpha}$ does not depend on the basis $e_{1}, \ldots, e_{n}$. Let $v_{1}, \ldots, v_{n}$ be another basis of $V$. Define a linear operator $T$ on $V$ by $T\left(e_{i}\right)=v_{i}, \quad l \leq i \leq n$, and linear extension. It is well known (see, e.g., [10]) that $T$ induces a linear operator $K(T)$ on $V_{\chi}(G)$ such that

$$
K(T)\left(x_{1} * \ldots * x_{m}\right)=\left(T x_{1}\right) * \ldots *\left(T x_{m}\right),
$$

for all $x_{1}, \ldots, x_{\mathrm{m}} \in V$. Since $T$ is invertible, it follows that $K(T)$ is invertible. Indeed, $K(T)^{-1}=$ $K\left(T^{-1}\right)$. Applying $K(T)$ to both sides of the equation $\mathrm{e}_{\alpha \sigma}^{*}=c_{\alpha}(\sigma) e_{\alpha}^{*}$ one obtains $v_{\alpha \sigma}^{*}=c_{\alpha}(\sigma) v_{\alpha}^{*}$.

THEOREM 1: For all $\alpha \in \Omega, \mathrm{G}^{\alpha}$ is a group and $c_{\alpha}$ is a linear character on it. 
Proof: If $\sigma, \pi \epsilon G$, then

$$
\begin{aligned}
e_{\alpha \sigma \pi}^{*} & =P\left(\pi^{-1}\right) e_{\alpha \sigma}^{*} \\
& =c_{\alpha}(\sigma) P\left(\pi^{-1}\right) e_{\alpha}^{*} \\
& =c_{\alpha}(\sigma) e_{\alpha \pi}^{*} \\
& =c_{\alpha}(\sigma) c_{\alpha}(\pi) e_{\alpha}^{*} .
\end{aligned}
$$

Thus $\sigma \pi \epsilon G^{\alpha}$ and, since $e_{\alpha}^{*} \neq 0, c_{\alpha}(\sigma \pi)=c_{\alpha}(\sigma) c_{\alpha}(\pi)$.

We remark (without proof since the result seems peripheral to the present undertaking) that the restriction of $\chi$ to $G^{\alpha}$ contains $c_{\alpha}$ as a component for all $\alpha \epsilon \Omega$, i.e.,

$$
(\chi, 1)_{G_{\alpha}}>0 \text { implies }\left(\chi, c_{\alpha}\right)_{G^{\alpha}}>0 .
$$

The converse fails.

Corollary 1: If $\alpha \in \Omega$, then $\mathrm{s}_{\alpha} \leq\left[\mathrm{G}: \mathrm{G}^{\alpha}\right]$. (Indeed, if $\mathrm{S}^{\alpha}$ is a system of right coset representatives for $\mathrm{G}^{\alpha}$ in $\mathrm{G}$, then $\left.\underset{\alpha \in \frac{1}{\Delta}}{\cup} \mathrm{e}_{\alpha \pi}^{*}: \quad \pi \in \mathrm{S}^{\alpha}\right\}$ spans $\mathrm{V}_{\chi}(\mathrm{G})$.)

(It follows from Corollary 1 and (iii) of section 1 that $\chi(i d) \leq\left[G: G^{\alpha}\right]$ for all $\alpha \in \Omega$. This inequality may be of some interest in itself because $G^{\alpha}$ is generally not normal in $G[1$, Theorem (53.17)].)

ExAmple 1: Let $G=S_{3}$. Let $\chi$ be the irreducible character of $G$ of degree 2, and take $\alpha=(1$, 1, 2). Then $G_{\alpha}=S_{2}$. If $G_{\alpha}$ were not all of $G^{\alpha}$, then $G^{\alpha}$ would be all of $S_{3}$, implying that $\left[G: G^{\alpha}\right]=$ $1<s_{\alpha}=2$, contradicting Corollary 1 . Therefore, $G_{\alpha}=G^{\alpha}$, and $\left[G: G^{\alpha}\right]=3$. In particular, it's not true in general that $s_{\alpha}=\left[\begin{array}{ll}G: & G^{\alpha}\end{array}\right]$.

Subsequent developments will make clearer the relationship between $G_{\alpha}$ and $G^{\alpha}$. We now make another definition. Let $G$ be a subgroup of $S_{m}$. Let $\chi$ be an irreducible character of $G$. Define

$$
G_{\chi}=\{\sigma \in G: \quad|\chi(\sigma)|=\chi(i d)\} .
$$

It is easy to see that $G_{\chi}$ is a normal subgroup of $G$ and $\lambda=\chi / \chi(i d)$ is a linear character on it [4, p. 35], [11]. In fact, $G_{\chi}$ consists of those $\sigma$ which are represented by scalars in any representation which affords $\chi$.

Theorem 2: For $\alpha \in \Omega, G \chi \subseteq \mathrm{G}^{\alpha}$, and the restriction of $\mathrm{c}_{\alpha}$ to $\mathrm{G}_{\chi}$ is $\lambda$.

Proof: Let $\sigma \in \mathrm{G} \chi$. Then

$$
\begin{aligned}
e_{\alpha \sigma}^{*} & =\frac{\chi(i d)}{o(G)} \sum_{\pi \in G} \chi(\pi) P\left(\pi \sigma^{-1}\right) e_{\alpha} \\
& =\frac{\chi(i d)}{o(G)} \sum_{\pi \in G} \chi(\pi \sigma) P(\pi) e_{\alpha}^{-} \\
& =\frac{\chi(i d)}{o(G)} \lambda(\sigma) \sum_{\pi \in G} \chi(\pi) P(\pi) e_{\alpha} \\
& =\lambda(\sigma) e_{\alpha}^{*} .
\end{aligned}
$$

Corollary 2: For all $\alpha \in \Omega, \mathrm{G}_{\alpha} \mathrm{G}_{\chi} \subseteq \mathrm{G}^{\alpha}$. 
EXAmple 2: It is tempting to conjecture that $G_{\alpha} G_{\chi}=G^{\alpha}$. Unfortunately, this is not always the case. Let $G=S_{5}$. Suppose $\chi$ arises from the frame $(3,2)$. Let $\alpha=(1,1,1,2,3)$ and let $\sigma$ be the transposition (45). Then $\alpha \sigma=(1,1,1,3,2)$, and a brute force computation shows that $e_{\alpha}^{*}=e_{\alpha \sigma}^{*}$. In particular, since $G_{\chi}=\{i d\}$ and $G_{\alpha}=S_{3}$, it follows that (45) $\epsilon G^{\alpha} \backslash G_{\alpha} G_{\chi}$.

It was proved in [11] that $\chi(i d)^{2} \leq\left[\mathrm{G}: G_{\chi}\right]$, so the inequality $s_{\alpha} \leq\left[\begin{array}{ll}G: & G_{\chi}\end{array}\right]$ which arises from Theorem 2 is not very interesting. However, one might be tempted to conjecture that $\chi(i d)^{2} \leq$ [G: $G_{\alpha} G_{\chi}$ ] for all $\alpha \epsilon \Omega$. A counterexample follows.

EXAMPLE 3. Let $G$ be the subgroup of $S_{4}$ generated by $\{(14)(23),(1234)\}$. Then $G$ is the dihedral group $D_{4}$ of order 8 . Let $\chi$ be the irreducible character of $G$ of degree 2 . Then $\chi(i d)=2=$ $-\chi((13)(24))$, and $\chi$ is zero on the rest of $G$. Thus, $G_{\chi}=\{i d$, (13)(24) $\}$. If $\alpha=(1,1,2,2)$, then $G_{\alpha}=$ $\{i d,(12)(34)\}$, and $\alpha \epsilon \bar{\Delta}$. Moreover, $G_{\alpha} G_{\chi}=\{i d,(12)(34),(13)(24),(14)(23)\}$ and $\left[G: G_{\alpha} G_{\chi}\right]=2$, which is less than $\chi(i d)^{2}=4$.

It is worth pointing out some other features of Example 3: Since

$$
1<s_{\alpha} \leq\left[\mathrm{G}: G^{\alpha}\right] \leq\left[G: G_{\alpha} G_{\chi}\right]=2,
$$

it follows that $s_{\alpha}=\left[\begin{array}{ll}G: & G_{\alpha} G_{\chi}\end{array}\right]$, and hence $G^{\alpha}=G_{\alpha} G_{\chi}$. Moreover, $\chi(i d)^{2}=\left[\begin{array}{ll}G: & G_{\chi}\end{array}\right]$. In a moment, we shall see that these observations are connected. First, however, it should be mentioned that the case of equality in $\chi(i d)^{2} \leq\left[\begin{array}{ll}G: & G_{\chi}\end{array}\right]$ is related to some recent work of F. DeMeyer, S. M. Gagola, G. Janusz, K. M. Timmer, and J. Yellen ([2], [3], [6], [13], and [14]) in which the case of equality in $\chi(i d)^{2} \leq[G: Z(G)]$ is studied. In particular, since $Z(G) \subseteq G_{\chi},[G: Z(G)]=\chi(i d)^{2}$ implies $\left[G: \quad G_{\chi}\right]=\chi(i d)^{2}$. $\alpha \in \Omega$.

THEOREM 3: If $\chi(i d)^{2}=\left[\begin{array}{ll}G: & G_{\chi}\end{array}\right]$, then $s_{\alpha}=\left[\begin{array}{ll}G: G_{\alpha} G_{\chi}\end{array}\right]$ (and therefore $G^{\alpha}=G_{\alpha} G_{\chi}$ ) for all D that

Proof: If $\left[G: G_{\chi}\right]=\chi(i d)^{2}$, then $\chi(\sigma) \neq 0$, if and only if $\sigma \in G_{\chi}$ [11]. It follows from Theorem

$$
\begin{aligned}
s_{\alpha} & =\frac{\chi(i d)}{o\left(G_{\alpha}\right)} \sum_{\sigma \in G_{\alpha}} \chi(\sigma) \\
& =\frac{\chi(i d)^{2}}{o\left(G_{\alpha}\right)} \underset{\sigma \in G_{\alpha} \cap G_{\chi}}{\searrow} \lambda(\sigma),
\end{aligned}
$$

where $\lambda=\chi / \chi(i d)$. Since $s_{\alpha} \neq 0$, and since $\lambda$ is a linear character, it must be that $\lambda(\sigma)=1$ for all $\sigma \epsilon G_{\alpha} \cap G_{\chi}$. Thus

$$
\begin{aligned}
s_{\alpha} & =\chi(i d)^{2} o\left(G_{\alpha} \cap G_{\chi}\right) / o\left(G_{\alpha}\right) \\
& =o(G) o\left(G_{\alpha} \cap G_{\chi}\right) / o\left(G_{\chi}\right) \circ\left(G_{\alpha}\right) \\
& =\left[G: \quad G_{\alpha} G_{\chi}\right]
\end{aligned}
$$

from elementary group theory (see, e.g., [7, p. 45]).

THEOREM 4: Let $\mathrm{e}_{1}, \ldots, \mathrm{e}_{\mathrm{n}}$ be an o.n. basis of $\mathrm{V}$. Let $\pi_{1}, \ldots, \pi_{\mathrm{k}}$ be right coset representatives for $\mathrm{G}_{\chi}$ in $\mathrm{G}$. Suppose $\chi(\mathrm{id})^{2}=\left[\mathrm{G}: \mathrm{G}_{\chi}\right]$. If $\alpha \in \Omega$ is such that $\mathrm{G}_{\alpha} \subseteq \mathrm{G}_{\chi}$, then $\left\{\mathrm{e}_{\alpha \pi_{1}}^{*}: 1 \leq \mathrm{i} \leq\right.$ $\left.\left[\mathrm{G}: \mathrm{G}_{\chi}\right]\right\}$ is an orthogonal basis of $\left\langle\mathrm{e}_{\alpha \sigma}^{*}: \sigma \in \mathrm{G}\right\rangle$.

Proof. Let $\lambda(\sigma)=\chi(\sigma) / \chi(i d), \sigma \in G_{\chi}$. Since $\alpha \epsilon \Omega$, it follows that $\lambda$ is. identically 1 on $G_{\alpha}$. 
Now,

$$
\begin{aligned}
\left(e_{\alpha \pi_{i}}^{*}, e_{\alpha \pi_{j}}^{*}\right) & =\left(e_{\alpha}^{*}, e_{\alpha \pi_{j} \pi_{i}-1}^{*}\right) \\
& =\frac{\chi(i d)}{\mathrm{o}(\boldsymbol{G})} \sum_{\tau \in G_{\alpha}} \chi\left(\pi_{i} \pi_{j}^{-1} \tau\right), \text { from (3) } \\
& =\frac{\chi(i d)}{\mathrm{o}(G)} \chi\left(\pi_{i} \pi_{j}^{-1}\right) \sum_{\tau \in G_{\alpha}} \lambda(\tau) \\
& =\chi(i d) \chi\left(\pi_{i} \pi_{j}^{-1}\right) /\left[G: \quad G_{\alpha}\right] .
\end{aligned}
$$

The result follows because $\chi\left(\pi_{i} \pi_{j}^{-1}\right) \neq 0$ if and only if $i=j$ (again appealing to [11]).

\section{References}

[ 1] Curtis, Charles, and Reiner, Irving, Representation Theory of Finite Groups and Associative Algebras (Interscience, New York, 1962).

[ 2] DeMeyer, Frank, Groups with an irreducible character of large degree are solvable, Proc. Amer. Math. Soc. 25, 615-617 (1970).

[ 3] DeMeyer, Frank, and Janusz, Gerald, Finite groups with an irreducible representation of large degree, Math. Z. $108,145-153$ (1969).

[ 4] Feit, Walter, Characters of Finite Groups (Benjamin, New York, 1967).

[ 5] Freese, Ralph, Inequalities for generalized matrix functions based on arbitrary characters, Linear Algebra Appl. 7, 337-34.5 (1973).

[ 6] Gagola, S. M., Characters fully ramified over a normal subgroup, preprint.

[ 7] Herstein, I. N., Topics in Algebra, 2nd. ed., (Xerox, Lexington MA, 1975).

[ 8] Marcus, Marvin, Finite Dimensional Multilinear Algebra, Part I, (Marcel Dekker, New York, 1973).

[ 9] Marcus, Marvin, and Minc, Henryk, Generalized matrix functions, Trans. Amer. Math. Soc. 1 16, $316-329$ (1965).

[10] Merris, Russell, and Pierce, Stephen, Elementary divisors of higher degree associated transformations, Linear and Multilinear Algebra 1, 241-250 ( 1973).

[11] Merris, Russell, and Watkins, William, Character induced subgroups, J. Res. Nat. Bur. Stand. (U.S.), 77B (Math, Sci.), Nos. 3\&4, 93-100 (1973).

[12] Pierce, Stephen, Orthogonal decompositions of tensor spaces, J. Res. Nat. Bur. Stand. (U.S.), 74B (Math. Sci.), No. 1, 41-44 (1970).

[13] Timmer, Kathleen, Subgroups of groups of central type, Trans. Amer. Math. Soc. 189, 133-162 (1974).

[14] Yellen, Jay, On solvability of certain groups of central type, Notices Amer. Math. Soc. 22, A-104 (1975).

(Paper 80B2-440) 